\title{
ON SOLVABILITY OF A BOUNDARY VALUE PROBLEM FOR AN INHOMOGENEOUS POLYHARMONIC EQUATION WITH A FRACTIONAL ORDER BOUNDARY OPERATOR
}

\section{B.KH. TURMETOV}

\begin{abstract}
In this paper we study the solvability of one boundary value problem for an inhomogeneous polyharmonic equation. As a boundary operator, we consider a differentiation operator of fractional order in the Hadamard sense. The considered problem is a generalization of the known Neumann problem.
\end{abstract}

Keywords: polyharmonic equation, fractional derivative, Neumann problems, Hadamard operators.

Mathematics Subject Classification: $35 \mathrm{~L} 75,35 \mathrm{Q} 53,37 \mathrm{~K} 10,37 \mathrm{~K} 35$

\section{INTRODUCTION}

Let $\Omega=\left\{x \in \mathbb{R}^{n}:|x|<1\right\}$ be the $n$-dimensional unit ball, $n \geqslant 2, \partial \Omega=\left\{x \in \mathbb{R}^{n}:|x|=1\right\}$ be the unit sphere. Let $u(x)$ be a smooth function in the ball $\Omega, r=|x|, \theta=x / r, \delta=r \frac{d}{d r}$ be the Dirac operator, where $\frac{d}{d r}=\sum_{j=1}^{n} \frac{x_{j}}{r} \frac{\partial}{\partial x_{j}}$.

For each $\alpha>0$, the following expression

$$
J^{\alpha}[u](x)=\frac{1}{\Gamma(\alpha)} \int_{0}^{r}\left(\ln \frac{r}{s}\right)^{\alpha-1} \frac{u(s \theta)}{s} d s
$$

is called the integration operator of order $\alpha$ in the Hadamard sense [1].

In what follows we assume that $J^{0}[u](x)=u(x)$.

By the change of variables $\xi=s r$, integral (1) is represented in the form

$$
J^{\alpha}[u](x)=\frac{1}{\Gamma(\alpha)} \int_{0}^{1}\left(\ln \frac{1}{\xi}\right)^{\alpha-1} \frac{u(\xi x)}{\xi} d \xi .
$$

We observe that the operator $J^{\alpha}$ can not be applied to continuous functions $u(x)$ as $u(0) \neq 0$, since the integral $\int_{0}^{1}\left(\ln \frac{1}{\xi}\right)^{\alpha-1} \xi^{-1} d \xi$ diverges. This is why for each $\alpha \in(\ell-1, \ell], \ell=1,2, \ldots$, as the differentiation operator of fractional order we consider the following modification of the Hadamard operator

B.Kh. Turmetov, On SOlvability of a boundary VAlue Problem For an INHOMOgeneous PolyHARMONIC EQUATION WITH A FRACTIONAL ORDER BOUNDARY OPERATOR.

(c) Turmetov B.Kh. 2016.

Thr work is supported by the grant no. 0819/GF4 of the Ministery of Education and Science of the Republic of Kazakhastan.

Submitted August 24, 2015. 


$$
D^{\alpha}[u](x)=J^{\ell-a}\left[\delta^{\ell}[u]\right](x) \equiv \frac{1}{\Gamma(\ell-\alpha)} \int_{0}^{r}\left(\ln \frac{r}{s}\right)^{\ell-1-\alpha}\left(s \frac{d}{d s}\right)^{\ell}[u](s \theta) \frac{d s}{s} .
$$

Let $0<\alpha \leqslant 1, m=1,2, \ldots$ In the domain $\Omega$, we consider the following problem

$$
\begin{aligned}
&(-\Delta)^{m} u(x)=f(x), \quad x \in \Omega, \\
& D^{\alpha+k}[u](x)=g_{k}(x), \quad x \in \partial \Omega, \quad k=0,1, \ldots, m-1 .
\end{aligned}
$$

A solution to problem (3), (4) is a function $u \in C^{2 m}(\Omega) \cap C(\bar{\Omega})$, for which $D^{\alpha+k}[u] \in C(\bar{\Omega})$, $k=0,1, \ldots, m-1$, and the equation (3) and boundary conditions (4) are satisfied in the classical sense.

We note that the boundary value problems for elliptic second order equations with fractional order boundary operators were studied in works [2]-[10]. The applications of boundary value problems for elliptic equations with fractional order boundary operators were considered in works [11]-[13]. The analogues of the Neumann problems for the biharmonic and polyharmonic equations in the case of the integer order boundary operators were studied in works [15]-[19], while boundary operators fractional in Riemann-Liouville, Caputo and Hadamard-Marchaud sense were addressed in [20]-23].

Since $J^{0}[u](x)=u(x)$, in the case $\alpha=1$ the operator $D^{1}$ coincides with the operator $\delta$, while $D^{k}=\delta^{k} \equiv\left(r \frac{d}{d r}\right)^{k}$. It was proved in [20] that the operator $\left(r \frac{d}{d r}\right)^{k}$ satisfies the identity

$$
\left(r \frac{\partial}{\partial r}\right)^{k}=\sum_{i=1}^{k}\left(\sum_{j=1}^{i}(-1)^{i-j} \frac{j^{n}}{j !(i-j) !}\right) r^{i} \frac{\partial^{i}}{\partial r^{i}} \equiv \sum_{i=1}^{k} a_{i}^{(k)} r^{i} \frac{\partial^{i}}{\partial r^{i}}, k=1,2, \ldots
$$

It is also known (see, for instance, [17]) that

$$
\left.r^{i} \frac{d^{i}}{d r^{i}}\right|_{\partial \Omega}=\left.r \frac{d}{d r}\left(r \frac{d}{d r}-1\right) \ldots\left(r \frac{d}{d r}-i+1\right)\right|_{\partial \Omega}=\left.\frac{\partial^{i} u(x)}{\partial \nu^{i}}\right|_{\partial \Omega}
$$

where $\nu$ is the outward normal for the boundary of the domain $\Omega$. Therefore, in the case $\alpha=1$, problem (3)-(4) is a some analogue of the Neumann problem for equation (3).

\section{Auxiliary statements}

In this section we study some properties of the operators $J^{\alpha}$ and $D^{\alpha}$.

Lemma 1. Let $0<\alpha, 0<\lambda<1$ and $u \in C^{\lambda+p}(\bar{\Omega}), p=0,1, \ldots$ If $u(0)=0$, then the function $J^{\alpha}[u](x)$ also belongs to the class $C^{\lambda+p}(\bar{\Omega})$ and the identity $J^{\alpha}[u](0)=0$ holds true.

Proof. Let $u(0)=0$. Then

$$
\left|J^{\alpha}[u](x)\right| \leqslant \frac{1}{\Gamma(\alpha)} \int_{0}^{1}\left(\ln \frac{1}{s}\right)^{\alpha-1} \frac{|u(s x)|}{s} d s \leqslant \frac{C}{\Gamma(\alpha)} \int_{0}^{1}\left(\ln \frac{1}{s}\right)^{\alpha-1} s^{\lambda-1} d s .
$$

Since the integral $\int_{0}^{1}\left(\ln \frac{1}{s}\right)^{\alpha-1} s^{\lambda-1} d s$ converges, then $\left|J^{\alpha}[u](x)\right| \leqslant C$, where $C \equiv$ const, and hence, the functiuon $J^{\alpha}[u](x)$ is well-defined. We denote $h(x)=J^{\alpha}[u](x)$. Then for each $x, y \in \bar{\Omega}$ we have

$$
|h(x)-h(y)| \leqslant \frac{1}{\Gamma(\alpha)} \int_{0}^{1}\left(\ln \frac{1}{s}\right)^{\alpha-1} \frac{|u(s x)-u(s y)|}{s} d s
$$




$$
\leqslant \frac{C|x-y|^{\lambda}}{\Gamma(\alpha)} \int_{0}^{1}\left(\ln \frac{1}{s}\right)^{\alpha-1} s^{\lambda-1} d s \leqslant C|x-y|^{\lambda} .
$$

In the same for each multi-index $\beta$ with $|\beta| \leqslant p$ we obtain

$$
\left|D^{\beta} h(x)-D^{\beta} h(y)\right| \leqslant \frac{1}{\Gamma(\alpha)} \int_{0}^{1}\left(\ln \frac{1}{s}\right)^{\alpha-1} \frac{\left|D^{\beta} u(s x)-D^{\beta} u(s y)\right|}{s} d s \leqslant C|x-y|^{\lambda} .
$$

Moreover,

$$
J^{\alpha}[u](0)=\lim _{x \rightarrow 0} J^{\alpha}[u](x)=\frac{1}{\Gamma(\alpha)} \int_{0}^{1}\left(\ln \frac{1}{s}\right)^{\alpha-1} s^{-1} \lim _{x \rightarrow 0} u(s x) d s=0 .
$$

In the same way we prove the following statement.

Lemma 2. Let $0<\alpha \leqslant 1,0<\lambda<1$ and $u \in C^{\lambda+p+1}(\bar{\Omega})$. Then $D^{\alpha}[u] \in C^{\lambda+p}(\bar{\Omega})$ and the identity $D^{\alpha}[u](0)=0$ holds true.

Lemma 3. Let $0<\alpha, \lambda<1$ and $u \in C^{\lambda+1}(\bar{\Omega})$. Then

1) for each $x \in \bar{\Omega}$, the identity

$$
J^{\alpha}\left[D^{\alpha}[u]\right](x)=u(x)-u(0)
$$

holds true;

2) if $u(0)=0$, then for each $x \in \bar{\Omega}$, the identity

$$
D^{\alpha}\left[J^{\alpha}[u]\right]=u(x)
$$

holds true.

Proof. If $u \in C^{\lambda+1}(\bar{\Omega})$, by Lemma 2.2 we obtain: $D^{\alpha}[u] \in C^{\lambda}(\bar{\Omega})$ and $D^{\alpha}[u](0)=0$. On the class of such functions, the operator $J^{\alpha}$ is well-defined and

$$
\begin{aligned}
J^{\alpha}\left[D^{\alpha}[u]\right](x) & =\frac{1}{\Gamma(\alpha)} \int_{0}^{r}\left(\ln \frac{r}{s}\right)^{\alpha-1} D^{\alpha}[u](s \theta) \frac{d s}{s} \\
& =\frac{1}{\Gamma(\alpha)} \frac{1}{\Gamma(1-\alpha)} \int_{0}^{r}\left(\ln \frac{r}{s}\right)^{\alpha-1} \int_{0}^{s}\left(\ln \frac{s}{\tau}\right)^{-\alpha} \delta[u](\tau \theta) \frac{d \tau}{\tau} \frac{d s}{s} \\
& =\frac{1}{\Gamma(\alpha)} \frac{1}{\Gamma(1-\alpha)} \int_{0}^{r} \frac{d u(\tau \theta)}{d \tau} \int_{s}^{r}\left(\ln \frac{r}{s}\right)^{\alpha-1}\left(\ln \frac{s}{\tau}\right)^{-\alpha} \frac{d s}{s} d \tau
\end{aligned}
$$

It is easy to show that

$$
\int_{s}^{r}\left(\ln \frac{r}{\tau}-\ln \frac{s}{\tau}\right)^{\alpha-1}\left(\ln \frac{s}{\tau}\right)^{-\alpha} d\left(\ln \frac{s}{\tau}\right)=\frac{\Gamma(\alpha) \Gamma(1-\alpha)}{\Gamma(1)} .
$$

Then

$$
J^{\alpha}\left[D^{\alpha}[u]\right](x)=\int_{0}^{r} \frac{d u(\tau \theta)}{d \tau} d \tau=u(x)-u(0)
$$

The identity (5) is proven. 
Since $u(0)=0$, by Lemma 2.1 the function $J^{\alpha}[u](x)$ is defined in domain $\bar{\Omega}$ and

$$
\begin{aligned}
D^{\alpha}\left[J^{\alpha}[u]\right](x) & =\frac{1}{\Gamma(1-\alpha)} \int_{0}^{r}\left(\ln \frac{r}{s}\right)^{-\alpha} s \frac{d}{d s} J^{\alpha}[u](s \theta) \frac{d s}{s} \\
& =\frac{1}{\Gamma(1-\alpha)} r \frac{d}{d r} \int_{0}^{r} \frac{1}{1-\alpha}\left(\ln \frac{r}{s}\right)^{1-\alpha} \frac{d}{d s} J^{\alpha}[u](s \theta) d s \\
& =\frac{1}{\Gamma(1-\alpha)} r \frac{d}{d r}\left[\left.\frac{1}{1-\alpha}\left(\ln \frac{r}{s}\right)^{1-\alpha} J^{\alpha}[u](s \theta)\right|_{s=0} ^{s=r}+\int_{0}^{r}\left(\ln \frac{r}{s}\right)^{-\alpha} J^{\alpha}[u](s \theta) d s\right]=r \frac{d}{d r}\left[J^{1-\alpha}\left[J^{\alpha}[u]\right]\right](x) . \\
& =r \frac{d}{d r}\left[\frac{1}{\Gamma(1-\alpha)} \int_{0}^{r}\left(\ln \frac{r}{s}\right)^{-\alpha} J^{\alpha}[u](s \theta) d s\right]
\end{aligned}
$$

Since $J^{1-\alpha} \cdot J^{\alpha}=J^{1}$, then

$$
D^{\alpha}\left[J^{\alpha}[u]\right](x)=r \frac{d}{d r} \int_{0}^{r} u(s \theta) \frac{d s}{s}=r \frac{u(r \theta)}{r}=u(x) .
$$

Lemma 4. Let $(-\Delta)^{m} u(x)=f(x)$, where $f(x)$ is a smooth function in $\bar{\Omega}$. Then the identity

$$
(-\Delta)^{m} D^{\alpha}[u](x)=F(x), \quad x \in \Omega,
$$

holds, where

$$
F(x)=|x|^{-2 m} D^{\alpha}\left[|x|^{2 m} f(x)\right] .
$$

Proof. Employing identity (2) for the function $D^{\alpha}[u](x)$, we have

$$
D^{\alpha}[u](x)=\frac{1}{\Gamma(1-\alpha)} \int_{0}^{1}\left(\ln \frac{1}{s}\right)^{-\alpha} s \frac{d}{d s}[u](s x) \frac{d s}{s} .
$$

It is easy to show that

$$
\Delta^{m}\left[s \frac{d}{d s}[u](s x)\right]=s^{2 m}\left(s \frac{d}{d s}+2 m\right) f(s x) .
$$

Moreover,

$$
s^{2 m}\left(s \frac{d}{d s}+2 m\right) f(s x)=s \frac{d}{d s}\left[s^{2 m} f(s x)\right] .
$$

Applying the operator $\Delta^{m}$ to the function $D^{\alpha}[u](x)$, we obtain

$$
\begin{aligned}
\Delta^{m} D^{\alpha}[u](x) & =\frac{1}{\Gamma(1-\alpha)} \int_{0}^{1}\left(\ln \frac{1}{s}\right)^{-\alpha} s \frac{d}{d s}\left[s^{2 m} f(s x)\right] \frac{d s}{s} \\
& =\frac{r^{-2 m}}{\Gamma(1-\alpha)} \int_{0}^{1}\left(\ln \frac{1}{s}\right)^{-\alpha} \frac{d}{d s}\left[(s r)^{2 m} f(s x)\right] d s=|x|^{-2 m} D^{\alpha}\left[|x|^{2 m} f(x)\right]=F(x) .
\end{aligned}
$$


Lemma 5. The function $F(x)$ can be represented as

$$
F(x)=\left(r \frac{d}{d r}+2 m\right) f_{1-\alpha}(x)
$$

where

$$
f_{1-\alpha}(x)=|x|^{-2 m} J^{1-\alpha}\left[|x|^{2 m} f(x)\right] .
$$

Proof. By the change of variables $s r=\xi$, it follows from representation (8) that

$$
\begin{aligned}
F(x) & =\frac{|x|^{-2 m}}{\Gamma(1-\alpha)} \int_{0}^{r}\left(\ln \frac{r}{\xi}\right)^{-\alpha} \frac{d}{d \xi}\left[\xi^{2 m} f(\xi \theta)\right] d \xi \\
& =\frac{|x|^{-2 m}}{\Gamma(1-\alpha)} r \frac{d}{d r}\left[\int_{0}^{r} \frac{1}{1-\alpha}\left(\ln \frac{r}{\xi}\right)^{1-\alpha} \frac{d}{d \xi}\left[\xi^{2 m} f(\xi \theta)\right] d \xi\right] \\
& =\frac{|x|^{-2 m}}{\Gamma(1-\alpha)} r \frac{d}{d r}\left[\left.\frac{1}{1-\alpha}\left(\ln \frac{r}{\xi}\right)^{1-\alpha} \xi^{2 m} f(\xi \theta)\right|_{\xi=0} ^{\xi=r}+\int_{0}^{r}\left(\ln \frac{r}{\xi}\right)^{-\alpha} \xi^{2 m} f(\xi \theta) \frac{d \xi}{\xi}\right] \\
& =|x|^{-2 m} r \frac{d}{d r}\left[\frac{1}{\Gamma(1-\alpha)} \int_{0}^{r}\left(\ln \frac{r}{\xi}\right)^{-\alpha} \xi^{2 m} f(\xi \theta) \frac{d \xi}{\xi}\right] \\
& =|x|^{-2 m} r \frac{d}{d r}\left[\frac{|x|^{2 m}}{\Gamma(1-\alpha)} \int_{0}^{1}\left(\ln \frac{1}{s}\right)^{-\alpha} s^{2 m} f(s x) \frac{d s}{s}\right] .
\end{aligned}
$$

We denote

$$
f_{1-\alpha}(x)=\frac{1}{\Gamma(1-\alpha)} \int_{0}^{1}\left(\ln \frac{1}{s}\right)^{-\alpha} s^{2 m} f(s x) \frac{d s}{s} .
$$

It is obvious that

$$
f_{1-\alpha}(x)=\frac{1}{\Gamma(1-\alpha)} \int_{0}^{1}\left(\ln \frac{1}{s}\right)^{-\alpha} s^{2 m} f(s x) \frac{d s}{s}=|x|^{-2 m} J^{1-\alpha}\left[|x|^{-2 m} f(x)\right] .
$$

Then

$$
F(x)=|x|^{-2 m} r \frac{d}{d r}\left[|x|^{2 m} f_{1-\alpha}(x)\right]=\left(r \frac{d}{d r}+2 m\right) f_{1-\alpha}(x),
$$

that is, identity (9) holds.

The following statement was proved in work [22].

Lemma 6. Let $0<\alpha \leqslant 1$, and $D^{\alpha+k} u(x), k=1,2, \ldots$ is well-defined. Then the identity

$$
D^{\alpha+k}[u](x)=\left(r \frac{d}{d r}\right)^{k} D^{\alpha}[u](x)
$$

holds true. 


\section{Study of Some Properties of SOlutions to Dirichlet Type PROBlem}

We consider the following problem

$$
\left\{\begin{array}{l}
(-\Delta)^{m} v(x)=F(x), \quad x \in \Omega, \\
\delta^{k}[v](x)=g_{k}(x), \quad x \in \partial \Omega, \quad k=0,1, \ldots, m-1 .
\end{array}\right.
$$

Assume that functions $V(x)$ and $w(x)$ solve the following problems:

$$
\begin{aligned}
& \left\{\begin{array}{l}
(-\Delta)^{m} V(x)=F(x), \quad x \in \Omega, \\
\delta^{k}[V](x)=0, \quad x \in \partial \Omega, \quad k=0,1, \ldots, m-1,
\end{array}\right. \\
& \left\{\begin{array}{l}
(-\Delta)^{m} w(x)=0, \quad x \in \Omega, \\
\delta^{k}[w](x)=g_{k}(x), \quad x \in \partial \Omega, \quad k=0,1, \ldots, m-1 .
\end{array}\right.
\end{aligned}
$$

Then $v(x)=V(x)+w(x)$.

Lemma 7. Let $0<\lambda<1$ and $F \in C^{\lambda+p-2 m}(\bar{\Omega}), p \geqslant 2 m$. Then

1) problem $(13)$ is uniquely solvable and the solution belongs to the class $C^{\lambda+p}(\bar{\Omega})$;

2) if the function $F(x)$ can be represented as

$$
F(x)=\left(r \frac{d}{d r}+2 m\right) g(x)
$$

then the identity

$$
V(0)=\frac{1}{4^{m-1}((m-1) !)^{2} \omega_{n}} \int_{\partial \Omega}\left(1-|y|^{2}\right)^{m-1} g(y) d \xi
$$

holds true.

Proof. In Section 1 we have shown that the boundary value problems in problem (13) are equivalent to the conditions

$$
\delta^{k}[V](x)=\sum_{i=0}^{k} a_{i}^{(k)} \frac{\partial^{i} V(x)}{\partial \nu^{i}}, \quad x \in \partial \Omega .
$$

Then problem (13) is equivalent to the Dirichlet problem for the equation $(-\Delta)^{m}=F(x)$, $x \in \Omega$. It is known (see, for instance, [24]) that for $F \in C^{\lambda+p-2 m}(\bar{\Omega})$, the Dirichlet problem is uniquely solvable in the class $C^{\lambda+p}(\bar{\Omega})$. The first statement of the lemma is proven.

It is also known (see, for instance, [15]) that the solution to the Dirichlet problem is represented as

$$
V(x)=\int_{\Omega} G_{m, n}(x, y) F(y) d y
$$

where $G_{m, n}(x, y)$ is the Green function of the Dirichlet problem.

We note that the function $G_{m, n}(x, y)$ was constructed explicitly in work [25]-[27]. For instance, it was shown in work [25] that $G_{m, n}(x, y)$ is of the form

$$
G_{m, n}(x, y)=K_{m, n}|x-y|^{2 m-n} \int_{1}^{a(x, y)}\left(t^{2}-1\right)^{m-1} t^{1-n} d t
$$

where

$$
a(x, y)=\frac{|x| y\left|-\frac{y}{|x|}\right|}{|x-y|}, \quad K_{m, n}=\frac{1}{4^{m-1}((m-1) !)^{2} n e_{n}}, \quad e_{n}=\frac{\pi^{n / 2}}{\Gamma\left(1+\frac{n}{2}\right)}
$$


Employing the identity $\omega_{n}=\frac{2 \pi^{n / 2}}{\Gamma\left(\frac{n}{2}\right)}$, the coefficient $K_{m, n}$ can be represented as

$$
K_{m, n}=\frac{1}{4^{m-1}((m-1) !)^{2} n} \frac{2 \frac{n}{2} \Gamma\left(\frac{n}{2}\right)}{2 \pi^{n / 2}}=\frac{1}{4^{m-1}((m-1) !)^{2} \omega_{n}} .
$$

In what follows we shall need the value of the function $G_{m, n}(x, y)$ at the point $x=0$. By the representation (18) we have

$$
G_{m, n}(0, y)=K_{m, n}|y|^{2 m-n} \int_{1}^{|y|^{-1}}\left(t^{2}-1\right)^{m-1} t^{1-n} d t .
$$

We denote the last integral by $I_{m, n}$. Let calculate this integral. If $n \neq 2(i+1), i=0,1, \ldots, m-$ 1 , then

$$
\begin{aligned}
I_{m, n} & =\int_{1}^{|y|^{-1}}\left(t^{2}-1\right)^{m-1} t^{1-n} d t=\sum_{i=0}^{m-1}(-1)^{m-1-i} C_{m-1}^{i} \int_{1}^{|y|^{-1}} t^{2 i+1-n} d t \\
& =\left.\sum_{i=0}^{m-1}(-1)^{m-1-i} C_{m-1}^{i} \frac{t^{2(i+1)-n}}{2(i+1)-n}\right|_{t=1} ^{t=|y|^{-1}} \\
& =\sum_{i=0}^{m-1}(-1)^{m-1-i} C_{m-1}^{i} \frac{1}{2(i+1)-n}\left[|y|^{n-2(i+1)}-1\right] .
\end{aligned}
$$

Let

$$
d_{m, n, i}=(-1)^{m-1-i} C_{m-1}^{i} \frac{1}{2(i+1)-n} .
$$

If $n$ takes one of the values $n=2(k+1), k=0,1, \ldots, m-1$, then

$$
\begin{aligned}
I_{m, n} & =\sum_{i=0, i \neq k}^{m-1}(-1)^{m-1-i} C_{m-1}^{i} \int_{1}^{|y|^{-1}} t^{2 i+1-n} d t+(-1)^{m-1-k} C_{m-1}^{k} \int_{1}^{|y|^{-1}} t^{-1} d t \\
& =\sum_{i=0, i \neq k}^{m-1} d_{m, n, i}\left[|y|^{n-2(i+1)}-1\right]+(-1)^{m-1-k} C_{m-1}^{k} \ln \frac{1}{|y|} .
\end{aligned}
$$

Then as $n \neq 2(i+1), i=0,1, \ldots, m-1$ we have

$$
G_{m, n}(0, y)=K_{m, n}\left[\sum_{i=0}^{m-1} d_{m, n, i}\left[|y|^{2 m-2(i+1)}-|y|^{2 m-n}\right]\right],
$$

while for other values $n$ we have

$$
\begin{aligned}
G_{m, n}(0, y)= & K_{m, n}\left[\sum_{i=0, i \neq k}^{m-1} d_{m, n, i}\left[|y|^{2 m-2(i+1)}-|y|^{2 m-n}\right]\right] \\
& +K_{m, n}(-1)^{m-1-k} C_{m-1}^{k}|y|^{2 m-n} \ln \frac{1}{|y|}
\end{aligned}
$$

Then in the case $n \neq 2(i+1), i=0,1, \ldots, m-1$, it follows from identity $(19)$ and representation (15) that

$$
V(0)=K_{m, n} \int_{\Omega}\left[\sum_{i=0}^{m-1} d_{m, n, i}\left[|y|^{2 m-2(i+1)}-|y|^{2 m-n}\right]\right]\left(\rho \frac{d}{d \rho}+2 m\right) g(y) d y .
$$


Passing to the spherical coordinates $y=(\rho, \xi)$, where $\xi$ are angular coordinates, we express the latter integral as

$$
\begin{aligned}
V(0) & =K_{m, n} \int_{|\xi|=1} \int_{0}^{1} \rho^{n-1}\left[\sum_{i=0}^{m-1} d_{m, n, i}\left[\rho^{2 m-2(i+1)}-\rho^{2 m-n}\right]\right]\left(\rho \frac{d}{d \rho}+2 m\right) g(\rho, \xi) d \rho d \xi \\
& =K_{m, n} \int_{|\xi|=1}\left[J_{1}(\rho, \xi)+J_{2}(\rho, \xi)\right] d \xi,
\end{aligned}
$$

where

$$
\begin{aligned}
& J_{1}(\rho, \xi)=\sum_{i=0}^{m-1} d_{m, n, i} \int_{0}^{1} \rho^{n-1}\left[\rho^{2 m-2(i+1)}-\rho^{2 m-n}\right] \rho \frac{d}{d \rho} g(\rho, \xi) d \rho, \\
& J_{2}(\rho, \xi)=2 m\left[\sum_{i=0}^{m-1} d_{m, n, i} \int_{0}^{1} \rho^{n-1}\left[\rho^{2 m-2(i+1)}-\rho^{2 m-n}\right] g(\rho, \xi) d \rho\right] .
\end{aligned}
$$

Integrating by parts in $J_{1}(\rho, \xi)$, we obtain

$$
\begin{aligned}
J_{1} & =\sum_{i=0}^{m-1} d_{m, n, i} \int_{0}^{1}\left[-(2 m+n-2(i+1)) \rho^{2 m+n-1-2(i+1)}+2 m \rho^{2 m-1}\right] g(\rho, \xi) d \rho= \\
& =\sum_{i=0}^{m-1} d_{m, n, i} \int_{0}^{1} \rho^{n-1}\left[-(2 m+n-2(i+1)) \rho^{2 m-2(i+1)}+2 m \rho^{2 m-n}\right] g(\rho, \xi) d \rho .
\end{aligned}
$$

Hence,

$$
\begin{aligned}
J_{1}(\rho, \xi)+J_{2}(\rho, \xi) & =\int_{0}^{1} \rho^{n-1} \sum_{i=0}^{m-1}(-1)^{m-1-i} C_{m-1}^{i} \rho^{2(m-i-1)} g(\rho, \xi) d \rho \\
& =\int_{0}^{1} \rho^{n-1}\left(1-\rho^{2}\right)^{m-1} g(\rho, \xi) d \rho .
\end{aligned}
$$

Therefore,

$$
\begin{aligned}
V(0) & =K_{m, n} \int_{|\xi|=1} \int_{0}^{1} \rho^{n-1}\left(1-\rho^{2}\right)^{m-1} g(\rho, \xi) d \rho d \xi \\
& =\frac{1}{4^{m-1}((m-1) !)^{2} \omega_{n}} \int_{\partial \Omega}\left(1-|y|^{2}\right)^{m-1} g(y) d \xi .
\end{aligned}
$$

If $n$ takes one of the values $n=2(k+1), k=0,1, \ldots, m-1$, as above, by identity (20) we obtain

$$
\begin{aligned}
v(0)= & K_{m, n}\left[\sum_{i=0, i \neq k}^{m-1} d_{m, n, i} \int_{\Omega}\left[|y|^{2 m-2(i+1)}-|y|^{2 m-n}\right]\left(\rho \frac{d}{d \rho}+2 m\right) g(y) d y\right] \\
& +K_{m, n}(-1)^{m-1-k} C_{m-1}^{k} \int_{\Omega}|y|^{2 m-n} \ln \frac{1}{|y|}\left(\rho \frac{d}{d \rho}+2 m\right) g(y) d y
\end{aligned}
$$




$$
=K_{m, n} \int_{|\xi|=1}\left[J_{1,1}(\rho, \xi)+J_{2,1}(\rho, \xi)\right] d \xi .
$$

Here

$$
\begin{aligned}
J_{1,1}(\rho, \xi)= & \sum_{i=0, i \neq k}^{m-1} d_{m, n, i} \int_{0}^{1} \rho^{n-1}\left[\rho^{2 m-2(i+1)}-\rho^{2 m-n}\right] \rho \frac{d}{d \rho} g(\rho, \xi) d \rho \\
& +(-1)^{m-1-k} C_{m-1}^{k} \int_{0}^{1} \rho^{n-1}\left(\rho^{2 m-n} \ln \frac{1}{\rho}\right) \rho \frac{d}{d \rho} g(\rho, \xi) d \rho \\
J_{2,1}(\rho, \xi)= & 2 m\left[\sum_{i=0}^{m-1} d_{m, n, i} \int_{0}^{1} \rho^{n-1}\left[\rho^{2 m-2(i+1)}-\rho^{2 m-n}\right] g(\rho, \xi) d \rho\right] \\
& +2 m(-1)^{m-1-k} C_{m-1}^{k} \int_{0}^{1} \rho^{n-1}\left(\rho^{2 m-n} \ln \frac{1}{\rho}\right) g(\rho, \xi) d \rho .
\end{aligned}
$$

Integrating by parts $J_{1,1}(\rho, \xi)$, we obtain

$$
\begin{aligned}
J_{1,1}= & \sum_{i=0, i \neq k}^{m-1} d_{m, n, i} \int_{0}^{1} \rho^{n-1}\left[-(2 m+n-2(i+1)) \rho^{2 m-2(i+1)}+2 m \rho^{2 m}\right] g(\rho, \xi) d \rho \\
& -(-1)^{m-1-k} C_{m-1}^{k} 2 m \int_{0}^{1} \rho^{n-1}\left(\rho^{2 m-n} \ln \frac{1}{\rho}\right) g(\rho, \xi) d \rho \\
& +(-1)^{m-1-k} C_{m-1}^{k} \int_{0}^{1} \rho^{n-1} \rho^{2 m-n} g(\rho, \xi) d \rho .
\end{aligned}
$$

Then

$$
\begin{aligned}
J_{1,1}(\rho, \xi)+J_{2,1}(\rho, \xi) & =\int_{0}^{1} \rho^{n-1} \sum_{i=0}^{m-1}(-1)^{m-1-i} C_{m-1}^{i} \rho^{2(m-i-1)} g(\rho, \xi) d \rho \\
& =\int_{0}^{1} \rho^{n-1}\left(1-\rho^{2}\right)^{m-1} g(\rho, \xi) d \rho .
\end{aligned}
$$

Hence, in this case we also have identity (16).

Let us study problem (14). Let $A$ be a matrix of

$$
A=\left(\begin{array}{crr}
* 20 c 11 & 1 \ldots & 1 \\
02 & 4 \ldots & 2(m-1) \\
02^{2} & 4^{2} \ldots & {[2(m-1)]^{2}} \\
: \vdots & \vdots & \vdots \\
02^{m-1} & 4^{m-1} & {[2(m-1)]^{m-1}}
\end{array}\right) .
$$


We denote by $\Delta_{j}, j=0,1, \ldots, m-1$ the determinant of the matrix obtained from the matrix $A$ by removing the first column and $j+1$ rows. In particular, $\Delta_{0}=|A|=\operatorname{det} A$. It is easy to show that $|A| \neq 0$.

Lemma 8. Let $0<\lambda<1$ and $g_{k} \in C^{\lambda+p-k}(\partial \Omega), p \geqslant m-1, k=0,1, \ldots, m-1$. Then

1) problem (14) is uniquely solvable and belongs to the class $C^{\lambda+p}(\bar{\Omega})$;

2) the solution to problem (14) satisfies the identity

$$
w(0)=-\frac{1}{\omega_{n}|A|} \sum_{j=0}^{m-1} \int_{\partial \Omega}(-1)^{j+1} \Delta_{j} \cdot g_{j}(x) d S_{x} .
$$

Proof. Let us show that problem (14) is equivalent to the Dirichlet problem for the equation $(-\Delta)^{m} w(x)=0$. In Section 1 we have shown that if $\nu$ is the outward normal for the sphere $\partial \Omega$, then for all $x \in \partial \Omega$ the identity

$$
\left.\frac{\partial^{k} w(x)}{\partial r^{k}}\right|_{\partial \Omega}=\left.r^{k} \frac{\partial^{k} w(x)}{\partial r^{k}}\right|_{\partial \Omega}=\left.\frac{\partial^{k} w(x)}{\partial \nu^{k}}\right|_{\partial \Omega}, \quad k=1,2, \ldots,
$$

holds true.

Then for $k=1$ we have $\delta[w](x)=r \frac{\partial w}{\partial r}$, and hence, it follows from the condition $\left.\delta[w](x)\right|_{\partial \Omega}=$ $g_{1}(x)$ that

For $k=2$ we have

$$
\left.\frac{\partial w}{\partial \nu}\right|_{\partial \Omega}=g_{1}(x) \equiv \varphi_{1}(x)
$$

$$
\delta^{2}[w](x)=\left(r \frac{\partial}{\partial r}\right)^{2} w(x)=r^{2} \frac{\partial^{2} w}{\partial r^{2}}+r \frac{\partial w}{\partial r} .
$$

Then by the boundary condition $\left.\delta^{2}[w]\right|_{\partial \Omega}=g_{2}$ we obtain

$$
\left.\frac{\partial^{2} w}{\partial \nu^{2}}\right|_{\partial \Omega}=g_{2}(x)-\left.\frac{\partial w}{\partial r}\right|_{\partial \Omega}=g_{2}(x)-g_{1} \equiv \varphi_{2}(x)
$$

In the general case, by employing the identity

$$
\delta^{k}[w](x)=\sum_{i=0}^{k} a_{i}^{(k)} \frac{\partial^{i} w(x)}{\partial \nu^{i}}, x \in \partial \Omega,
$$

we obtain

$$
\delta^{k}[w](x)=r^{k} \frac{\partial^{k} w}{\partial r^{k}}+\sum_{i=1}^{k-1} a_{i}^{(k)} r^{i} \frac{\partial^{i} w}{\partial r^{i}} .
$$

Employing the boundary conditions $\left.\delta^{k}[w](x)\right|_{\partial \Omega}=g_{k}(x)$ in problem (14), for $\frac{\partial^{k} v}{\partial \nu^{k}}(x)$ we obtain

$$
\left.\frac{\partial^{k} v}{\partial \nu^{k}}\right|_{\partial \Omega}=g_{k}(x)-\sum_{i=0}^{k-1} b_{i, k} g_{i}(x) \equiv \varphi_{k}(x), k=2,3, \ldots, m-1,
$$

where the coefficients $b_{i, k}$ depend on $a_{i}^{(k)}, i<k$. Thus, problem (14) is equivalent to the Dirichlet problem:

$$
\left\{\begin{array}{l}
\Delta^{m} w(x)=0 \\
\left.\frac{\partial^{k} w}{\partial \nu^{k}}(x)\right|_{\partial \Omega}=\varphi_{k}(x), \quad k=0,1, \ldots, m-1 .
\end{array}\right.
$$

It is obvious that as $g_{k} \in C^{\lambda+p-k}(\partial \Omega)$, the functions $\varphi_{k}(x)$ also belong to the class $C^{\lambda+p-k}(\partial \Omega)$. Then by the known statement for the Dirichlet problem [24] it follows that 
problem (14) is uniquely solvable and the solution belongs to the class $C^{\lambda+p}(\bar{\Omega})$. The first of the lemma is proven.

We proceed to the proof of the second statement. Let $w(x)$ be the solution to problem (14). Since the function $w(x)$ is polyharmonic, there exist the harmonic in $\Omega$ functions $w_{j}(x)$, $j=\overline{0, m-1}$, such that

$$
w(x)=w_{0}(x)+|x|^{2} w_{1}(x)+\ldots+|x|^{2(m-1)} w_{m-1}(x) .
$$

We apply the operator $\left(r \frac{\partial}{\partial r}\right)^{\ell}, \ell=1,2, \ldots, m-1$, to the functions of the form $|x|^{2 j} w_{j}(x)$. Then for all $1 \leqslant \ell \leqslant m-1$ and $0 \leqslant j \leqslant m-1$ we find

$$
\begin{aligned}
\left(r \frac{\partial}{\partial r}\right)^{\ell}\left[|x|^{2 j} w_{j}(x)\right] & =\sum_{i=1}^{\ell} a_{i}^{(\ell)} r^{i} \frac{\partial^{i}}{\partial r^{i}}\left[r^{2 j} w_{j}(x)\right] \\
& =\sum_{i=1}^{\ell} a_{i}^{(\ell)} r^{i} \sum_{p=0}^{i} C_{i}^{p} \frac{\partial^{p} r^{2 j}}{\partial r^{p}} \frac{\partial^{i-p} w_{j}(x)}{\partial r^{i-p}}=\sum_{i=1}^{\ell} a_{i}^{(\ell)} r^{i} \sum_{p=0}^{i} C_{i}^{p} d_{i, j}^{(p)} r^{2 j-p} \frac{\partial^{i-p} w_{j}(x)}{\partial r^{i-p}},
\end{aligned}
$$

where

$$
d_{i, j}^{(p)}=\left\{\begin{aligned}
0, & p>2 j \\
1, & p=0 \\
2 j(2 j-1) \ldots(2 j-p+1), & p \leqslant 2 j .
\end{aligned}\right.
$$

Thus, the function $\left(r \frac{\partial}{\partial r}\right)^{\ell}\left[r^{2 j} w_{j}(x)\right]$ can be represented as

$$
\left(r \frac{\partial}{\partial r}\right)^{\ell}\left[|x|^{2 j} w_{j}(x)\right]=|x|^{2 j} h_{j, \ell}(x)
$$

where

$$
h_{j, l}(x)=\sum_{i=1}^{\ell} a_{i}^{(\ell)} \sum_{p=0}^{i} C_{i}^{p} d_{i, j}^{(p)} r^{i-p} \frac{\partial^{i-p} w_{j}(x)}{\partial r^{i-p}} .
$$

Since for harmonic function $w_{j}(x)$, the functions $r^{i-p} \frac{\partial^{i-p} w_{j}(x)}{\partial r^{i-p}}$ are also harmonic $\Omega$, then for all $j=0, \ldots, m-1, \ell=1, \ldots, m-1$ the functions $h_{j, \ell}(x)$ are harmonic in $\Omega$. On the other hand, expanding the functions $w_{j}(x), j=\overline{0, m-1}$ into the series of the form

$$
w_{j}(x)=\sum_{k=0}^{\infty} \sum_{i=1}^{h_{k}} w_{k, j}^{(i)} H_{k}^{(i)}(x)
$$

and applying the operator $\left(r \frac{\partial}{\partial r}\right)^{\ell}, \ell=1,2, \ldots, m-1$ to the function $|x|^{2 j} w_{j}(x)$ for all $x \in \Omega$, we obtain

$$
\left(r \frac{\partial}{\partial r}\right)^{\ell}\left[|x|^{2 j} w_{j}(x)\right]=|x|^{2 j} \sum_{k=0}^{\infty} \sum_{i=1}^{h_{k}}(k+2 j)^{\ell} w_{k, j}^{(i)} H_{k}^{(i)}(x)=|x|^{2 j} h_{j, \ell}(x) .
$$

Therefore, as $|x|<1$, the representation

$$
h_{\ell, j}(x)=\sum_{k=0}^{\infty} \sum_{i=1}^{h_{k}}(k+2 j)^{\ell} w_{k, j}^{(i)} H_{k}^{(i)}(x)
$$

holds true.

Thus, for the polyharmonic function $\left(r \frac{\partial}{\partial r}\right)^{\ell} w(x)$ we obtain

$$
\left(r \frac{\partial}{\partial r}\right)^{l} w(x)=h_{0, l}(x)+|x|^{2} h_{i, e}(x)+\ldots+|x|^{2(m-1)} h_{m-1, l}(x),
$$


where the harmonic functions $h_{j, \ell}(x)$ are determined by the identity (3.14) and are expanded into the series of form (26).

We consider harmonic in $\Omega$ functions

$$
\left\{\begin{array}{l}
z_{0}(x)=w_{0}(x)+w_{1}(x)+\ldots+w_{m-1}(x) \\
z_{\ell}(x)=h_{0, \ell}(x)+h_{1, \ell}(x)+\ldots+h_{m-1, \ell}(x), \quad \ell=1,2, \ldots, m-1 .
\end{array}\right.
$$

It is obvious that

$$
\left.z_{\ell}(x)\right|_{\partial \Omega}=h_{0, \ell}(x)+h_{1, \ell}(x)+\ldots+\left.h_{m-1, \ell}(x)\right|_{\partial \Omega}=g_{\ell}(x), \quad \ell=\overline{0, m-1} .
$$

Then the functions $z_{\ell}(x)$ can be represented as the Poisson integral

$$
z_{\ell}(x)=\frac{1}{\omega_{n}} \int_{\partial \Omega} \frac{1-|x|^{2}}{|x-y|^{n}} g_{\ell}(y) d S_{y} .
$$

Hence, for each $\ell=0,1, \ldots, m-1$

$$
z_{\ell}(0)=\frac{1}{\omega_{n}} \int_{\partial \Omega} g_{\ell}(y) d S_{y}
$$

It follows from expansion $(26)$ that

$$
h_{\ell, j}(0)=\sum_{\ell=1}^{h_{0}}(2 j)^{\ell} w_{0, j}^{(i)} H_{0}^{(i)}=(2 j)^{\ell} w_{j}(0), \ell \geqslant 1 .
$$

Then by identities (27) we obtain the system of equations

$$
\left\{\begin{array}{c}
w_{0}+w_{1}+\cdots+w_{m-1}=z_{0} \\
0 \cdot w_{0}+2 \cdot w_{1}+\cdots+2(m-1) \cdot w_{m-1}=z_{1} \\
\cdots \\
0 \cdot w_{0}+2^{m-1} \cdot w_{1}+\cdots+[2(m-1)]^{m-1} \cdot w_{m-1}=z_{m-1} .
\end{array}\right.
$$

The matrix of this system is of form (21). It follows from representation $(23)$ that $w(0)=w_{0}(0)$.

Let us show that the value $w_{0}(0)$ is expressed via linear combinations of the integrals of the functions $g_{j}(x), j=\overline{0, m-1}$ over the sphere $\partial \Omega$. Indeed, since $|A|=\operatorname{det} A \neq 0$, by the Cramer rule, we find the value $w_{0}(0)$ from the system 29$)$ by the formula

$$
w_{0}(0)=\frac{\Delta_{z}}{|A|}
$$

where $\Delta_{z}$ stands for the determinant

$$
\Delta_{z}=\left|\begin{array}{ccccc}
z_{0}(0) & 1 & 1 & \ldots & 1 \\
z_{1}(0) & 2 & 4 & \ldots & 2(m-1) \\
z_{2}(0) & 2^{2} & 4^{2} & \ldots & {[2(m-1)]^{2}} \\
\vdots & \vdots & \vdots & & \vdots \\
z_{m-1}(0) & 2^{m-1} & 4^{m-1} & \ldots & {[2(m-1)]^{m-1}}
\end{array}\right|
$$

It is obvious that $\Delta_{z}=-\sum_{j=0}^{m-1}(-1)^{j+1} \Delta_{j} \cdot z_{j}(0)$. And since $z_{j}(0)$ are determined by (28), then

$$
\Delta_{z}=-\frac{1}{\omega_{n}} \sum_{j=0}^{m-1} \int_{\partial \Omega}(-1)^{j+1} \Delta_{j} \cdot g_{j}(x) d S_{x}
$$


Now it follows from identity $(30)$ that

$$
w(0)=w_{0}(0)=\frac{\Delta_{z}}{|A|}=-\frac{1}{\omega_{n}|A|} \sum_{j=0}^{m-1} \int_{\partial \Omega}(-1)^{j+1} \Delta_{j} \cdot g_{j}(x) d S_{x} .
$$

Hence, identity (22) holds true.

Lemmata 3.1 and 3.2 imply the following statement.

Lemma 9. Let $0<\lambda<1, g_{k} \in C^{\lambda+p-k}(\partial \Omega), k=0,1, \ldots, m-1$, and $F \in C^{\lambda+p-2 m}(\bar{\Omega})$. Then

1) problem $(12)$ is uniquely solvable and the solution belongs to the class $C^{\lambda+p}(\bar{\Omega})$;

2) if the function $F(x)$ is represented as $(15)$, then the identity $v(0)=0$ is necessary and sufficient for the condition

$$
\int_{\Omega}\left(1-|y|^{2}\right)^{m-1} g(y) d \xi=\frac{4^{m-1}((m-1) !)^{2}}{|A|} \sum_{j=0}^{m-1} \int_{\partial \Omega}(-1)^{j+1} \Delta_{j} \cdot g_{j}(x) d S_{x} .
$$

\section{MAin STATEMENT}

In this section we provide the main statement on problem (3), (4).

We have the following statement.

Theorem 1. Let $0<\alpha \leqslant 1,0<\lambda<1, g_{k} \in C^{\lambda+m-1-k}(\partial \Omega), k=0,1, \ldots, m-1$ and $f \in C^{\lambda+1}(\bar{\Omega})$. Then problem (3), (4) is solvable if and only if

$$
\int_{\Omega}\left(1-|y|^{2}\right)^{m-1} f_{1-\alpha}(y) d y=\sum_{j=0}^{m-1} \int_{\partial \Omega} a_{j, m} g_{j}(x) d S_{x},
$$

where

$$
f_{1-\alpha}(x)=|x|^{-2 m} J^{1-\alpha}\left[|x|^{2 m} f(x)\right], a_{j, m}=\frac{4^{m-1}((m-1) !)^{2}}{|A|} \cdot(-1)^{j+1} \Delta_{j},
$$

$|A|$ is the determinant of the matrix $A$ in identity (21), $\Delta_{j}, j=0,1, \ldots, m-1$, are the determinants of the matrices obtained from the matrix $A$ by removing the first column and $j+1$-th row.

If the problem is solvable, the solution belongs to the class $C^{\lambda+m-1}(\bar{\Omega})$ and is unique up to a constant term. It is represented as

$$
u(x)=C+J^{\alpha}[v](x),
$$

where $v(x)$ is the solution to problem (12) with the function $F(x)=|x|^{-2 m} D^{\alpha}\left[|x|^{2 m} f(x)\right]$ satisfying the additional condition $v(0)=0$.

Proof. Assume that problem (3)-(4) exists and it is $u(x)$. We apply the operator $D^{\alpha}$ to the function $u(x)$ and denote $v(x)=D^{\alpha}[u]$. Let us find the conditions for the function $v(x)$. First of all, by Lemma 2.2, the identity $v(0)=0$ holds true. By Lemma 2.5, the representation (11) holds, that is,

Then

$$
D^{\alpha+k}[u](x)=\left(r \frac{d}{d r}\right)^{k}\left[D^{\alpha}[u]\right](x) \equiv \delta^{k}[v](x) .
$$

$$
\left.v(x)\right|_{\partial \Omega}=\left.D^{\alpha}[u](x)\right|_{\partial \Omega}=g_{0}(x),\left.\quad \delta^{k}[v](x)\right|_{\partial \Omega}=\left.D^{\alpha+k}[u](x)\right|_{\partial \Omega}=g_{k}(x), \quad k=1,2, \ldots
$$

And finally, applying the operator $(-\Delta)^{m}$ to the identity $v(x)=D^{\alpha}[u](x)$, by formula (7) in Lemma 2.3 we obtain

$$
(-\Delta)^{m} v(x)=(-\Delta)^{m} D^{\alpha}[u](x)=F(x),
$$


where $F(x)$ is defined by identity (8).

Thus, if $u(x)$ is a solution to problem (3), (4), then for the function $v(x)=D^{\alpha}[u](x)$ we obtain the problem (12) with the function $F(x)=|x|^{-2 m} D^{\alpha}\left[|x|^{2 m} f\right](x)$. Moreover, since $D^{\alpha}[u](0)=0$, the function $v(x)$ should satisfy the condition $v(0)=0$. Under the assumptions of the theorems, by Lemma 3.3 problem $(12)$ is uniquely solvable and the solution belongs to the class $C^{\lambda+m-1}(\bar{\Omega})$. In order to satisfy the condition $v(0)=0$, we need condition $(31)$, which in our case has form $(32)$.

Therefore, if problem (3), (4) is solvable, then condition (32) is satisfied. Let us show that this condition is also sufficient for the solvability of problem (3), (4).

Indeed, assume that in problem (12), the function $F(x)$ can be represented as $F(x)=$ $|x|^{-2 m} D^{\alpha}\left[|x|^{2 m} f\right](x)$. Then under the condition $f \in C^{\lambda+1}(\bar{\Omega})$ we have $F \in C^{\lambda}(\bar{\Omega})$ and if $g_{k} \in C^{\lambda+m-1-k}(\partial \Omega), k=0,1, \ldots, m-1$, then by Lemma 3 problem $(12)$ is uniquely solvable and the solution belongs to the class $C^{\lambda+m-1}(\bar{\Omega})$. If condition $(32)$ is satisfied, this solution also satisfies the condition $v(0)=0$. This is why in the class of such functions the operator $J^{\alpha}$ is well-defined and therefore, we can consider the function $C+J^{\alpha}[v](x)$. We denote this function by $u(x)$, that is, we consider the function $u(x)=C+J^{\alpha}[v](x)$. Let us show that this function satisfies all the conditions in problem (3), (4).

Indeed, since $v(0)=0$, by Lemma 2.1 the function $J^{\alpha}[v](x)$ belongs to the class $C^{\lambda+m-1}(\bar{\Omega})$. Applying the operator $(-\Delta)^{m}$ to this function, we obtain

$$
\begin{aligned}
(-\Delta)^{m} u(x) & =(-\Delta)^{m}[C]+(-\Delta)^{m} J^{\alpha}[v](x) \\
& =\frac{1}{\Gamma(\alpha)} \int_{0}^{1}\left(\ln \frac{1}{s}\right)^{\alpha-1} s^{2 m-1}(-\Delta)^{m} v(s x) d s \\
& =\frac{1}{\Gamma(\alpha)} \int_{0}^{1}\left(\ln \frac{1}{s}\right)^{\alpha-1} s^{2 m} F(s x) \frac{d s}{s} \\
& =\frac{1}{\Gamma(\alpha)} \int_{0}^{1}\left(\ln \frac{1}{s}\right)^{\alpha-1} s^{2 m}|s x|^{-2 m} D^{\alpha}\left[|x|^{2 m} f\right](s x) \frac{d s}{s} \\
& =|x|^{-2 m} J^{\alpha}\left[D^{\alpha}\left[|x|^{2 m} f\right]\right](x) .
\end{aligned}
$$

Then, by identity (5),

$$
J^{\alpha}\left[D^{\alpha}\left[|x|^{2 m} f\right]\right](x)=|x|^{2 m} f(x)-\left.|x|^{2 m} f(x)\right|_{x=0}=|x|^{2 m} f(x) .
$$

Then $(-\Delta)^{m} J^{\alpha}[v](x)=f(x)$, that is, the function $u(x)$ satisfies equation (3). Moreover, it follows from condition (6) that

$$
D^{\alpha}[u](x)=D^{\alpha}\left[J^{\alpha}[v]+C\right](x)=D^{\alpha}\left[J^{\alpha}[v]\right](x)=v(x) .
$$

Therefore,

$$
\begin{aligned}
& \left.D^{\alpha}[u](x)\right|_{\partial \Omega}=\left.v(x)\right|_{\partial \Omega}=g_{1}(x), \\
& \left.D^{\alpha+k}[u](x)\right|_{\partial \Omega}=\left.\left(r \frac{d}{d r}\right)^{k} D^{\alpha}[u](x)\right|_{\partial \Omega}=\left.\left(r \frac{d}{d r}\right)^{k} v(x)\right|_{\partial \Omega}=g_{k}(x), \quad k=1,2, \ldots
\end{aligned}
$$

Let us consider the solvability conditions of problem (3), (4) for some particular cases. 
Example 1. If $m=1$, we obtain the boundary value problem for the Poisson equation. Since in this case $|A|=\Delta_{0}=1$, the solvability condition of this problem is of the form:

$$
\int_{\Omega} f_{1-\alpha}(y) d y=\int_{\partial \Omega} g_{0}(y) d S_{y} .
$$

If $\alpha=1$, then $f_{0}(y) \equiv f(y)$ and we obtain the solvability condition of the classical Neumann problem for the Poisson equation.

Example 2. Let $m=2$, then

$$
\begin{gathered}
A=\left(\begin{array}{ll}
1 & 1 \\
0 & 2
\end{array}\right), \quad|A|=2, \quad \Delta_{z}=\left|\begin{array}{ll}
z_{0} & 1 \\
z_{1} & 2
\end{array}\right|, \quad \Delta_{0}=2, \quad \Delta_{1}=1, \\
a_{0,2}=\frac{4}{2} \cdot(-1) \Delta_{0}=-4, a_{1,2}=\frac{4}{2} \cdot(-1)^{2} \Delta_{1}=2 .
\end{gathered}
$$

Then the solvability condition of the problem is of the form

$$
\int_{\Omega} \frac{\left(1-|y|^{2}\right)}{2} f_{1-\alpha}(y) d y=\int_{\partial \Omega}\left[g_{1}(y)-2 g_{0}(y)\right] d S_{x} .
$$

\section{BIBLIOGRAPHY}

1. A.Kilbas, H. Srivastava, J. Trujillo. Theory and applications of fractional differential equations Elsevier, North-Holland (2006).

2. A.S. Berdyshev, B.Kh. Turmetov, B. J. Kadirkulov. Some properties and applications of the integrodifferential operators of Hadamard-Marchaud type in the class of harmonic functions // Sibir. Matem. Zhurn. 53:4, 752-764. [Siberian Math. J. 53:4, 600-610 (2012).]

3. V.Karachik, B.Turmetov, B.Torebek. On some integro-differential operators in the class of harmonic functions and their applications // Siber. Adv. Math. 22:2, 115-134 (2012).

4. M. Kirane, N.-E. Tatar. Absence of local and global solutions to an elliptic system with timefractional dynamical boundary conditions // Sibir. Matem. Zhurn. 48:3, 593-605 (2007). [Siber. Math. J. 48:3, 477-488 (2007).]

5. M. Kirane, N.-E. Tatar. Nonexistence for the Laplace equation with a dynamical boundary condition of fractional type // Sibir. Matem. Zhurn. 48:5, 1056-1064 (2007). [Siber. Math. J. 48:5, 849-856 (2007).]

6. M.Krasnoschok, N. Vasylyeva. On a nonclassical fractional boundary-value problem for the Laplace operator // J. Diff. Equat. 257:6, 1814-1839 (2014).

7. B. Torebek, B. Turmetov. On solvability of a boundary value problem for the Poisson equation with the boundary operator of a fractional order // Bound. Value Prob. 2013, id 93, (2013).

8. B.Kh. Turmetov. A boundary value problem for the harmonic equation // Differ. Uravn. 32:8, 1089-1092 (1996). [Differ. Equat. 32:8, 1093-1096 (1996).]

9. B.Kh. Turmetov. On smoothness of a solution to a boundary value problem with fractional-order boundary operator // Matem. Trudy. 7:1, 189-199 (2004) [Siber. Adv. Math. 15:2, 115-125 (2005).]

10. B.Kh. Turmetov, B.T. Torebek. Modified Bavrin operators and their applications // Differ. Uravn. 51:2, 240-250 (2015). [Differ. Equat. 51:2, 243-254 (2015).]

11. T. Akhmedov, E. Veliev, M. Ivakhnychenko. Fractional operators approach in electromagnetic wave reflection problems // J. Electromagnetic Waves Appl. 21:13, 1787-1802 (2007).

12. T. Akhmedov, E. Veliev, M. Ivakhchenko. Boundary conditions with fractional derivatives in the problems in the diffraction theory // Dokl. NAN Azerbaydzhana 65:2, 61-68 (2009). (in Russian).

13. E. Veliev, M. Ivakhnychenko. Fractional boundary conditions in plane wave diffraction on a strip // Progress in Electromagnetics Research. 79, 443-462 (2008).

14. A.V. Bitsadze. Some properties of polyharmonic functions // Differ. Uravn. 24:5, 825-831 (1988). [Differ. Equat. 24:5, 543-548 (1988).] 
15. B. Kanguzhin, B. Koshanov. Necessary and sufficient conditions of solvability of boundary value problems for inhomogeneous polyharmonic equation in a ball // Ufimskij Matem. Zhurn. 2:2, 41-52 (2010).

16. V. Karachik, B. Turmetov, A. Bekayeva. Solvability conditions of the Neumann boundary value problem for the biharmonic equation in the unit ball // Int. J. Pure Appl. Math. 81:3, 487-485 (2012).

17. V. Karachik. Solvability conditions for the Neumann problem for the homogeneous polyharmonic equation // Differ. Equat. 50:11, 1449-1456 (2014).

18. V. Karachik. On solvability conditions for the Neumann problem for a polyharmonic equation in the unit ball // J. Appl. Industrial Math. 8:1, 63-75 (2014).

19. B. Turmetov, R. Ashurov. On solvability of the Neumann boundary value problem for a nonhomogeneous polyharmonic equation in a ball // Boundary Value Problems. 2013, id 162 (2013).

20. A.E. Bekaeva, V.V. Karachik, B.Kh. Turmetov. Solvability of some boundary-value problems for polyharmonic equation with Hadamard-Marchaud boundary operator // Izv. Vyssh. Ucheb. Zaved. Matem. 7, 15-29 (2014). [Russ. Math. Izv. VUZ. Matem. 58:7, 11-24 (2014).]

21. A. Berdyshev, A. Cabada, B. Turmetov. On solvability of a boundary value problem for a nonhomogeneous biharmonic equation with a boundary operator of a fractional order // Acta Math. Scientia. Ser. B. 34:6, 1695-1706 (2014).

22. A. Berdyshev, A. Cabada, B. Turmetov. On solvability of some boundary value problem for polyharmonic equation with boundary operator of a fractional order // Appl. Math. Modelling. 39:15, 4548-4569 (2015).

23. B. Turmetov. Solvability of fractional analogues of the Neumann problem for a nonhomogeneous biharmonic equation // Elect. J. Diff. Equat. 2015:82, 1-21 (2015).

24. S.Agmon, A. Duglas, L. Nirenberg. Estimates near the boundary for elliptic partial. differential equations satisfying general boundary conditions. I. // Comm. Pure Appl. 12:12, 623-727 (1959).

25. F. Gazzola, H.-Ch. Grunau, S. Guido. Polyharmonic boundary value problems. Springer, Berlin (2010).

26. T.S. Kal'menov, D. Suragan. On a new method for constructing the green function of the Dirichlet problem for the polyharmonic equation // Differ. Equat. 48:3, 441-445 (2012).

27. T. Kal'menov, B. Koshanov, M. Nemchenko. Green function representation for the Dirichlet problem of the polyharmonic equation in a sphere // Compl. Var. Ellipt. Equat. 53:2, 177-183 (2008).

Batirkhan Khudaibergenovich Turmetov,

Institute of mathematics and mathematical modeling,

Pushkin str. 125,

050010, Almaty, Kazakhstan,

Kh. Yasavi International Kazakh-Turkish University,

B. Sattarkhanov str. 29,

161200, Turkestan, Kazakhstan

E-mail: turmetovbh@mail.ru 